\title{
High wall shear stress beyond a certain range in the parent artery could predict the risk of anterior communicating artery aneurysm rupture at follow-up
}

\author{
Xin Zhang, MD, ${ }^{1}$ Tamrakar Karuna, MD, ${ }^{2}$ Zhi-Qiang Yao, MD, ${ }^{1,3}$ Chuan-Zhi Duan, MD, ${ }^{1}$ \\ Xue-Min Wang, PhD, ${ }^{4}$ Shun-Ting Jiang, PhD, ${ }^{5}$ Xi-Feng Li, PhD, ${ }^{1}$ Jia-He Yin, MD, ${ }^{1}$ Xu-Ying He, MD, ${ }^{1}$ \\ Shen-Quan Guo, PhD, ${ }^{1}$ Yun-Chang Chen, PhD, ${ }^{1}$ Wen-Chao Liu, PhD, ${ }^{1}$ Ran Li, PhD, ${ }^{1}$ and \\ Hai-Yan Fan, PhD'
}

\begin{abstract}
${ }^{1}$ National Key Clinical Specialty/Engineering Technology Research Center of Education Ministry of China, Guangdong Provincial Key Laboratory on Brain Function Repair and Regeneration, Neurosurgery Institute, Department of Neurosurgery, Zhujiang Hospital, Southern Medical University, Guangzhou, China; ' $D$ epartment of Neurosurgery, CMS-Teaching Hospital, Bharatpur, Chitwan, Nepal; ${ }^{3}$ Department of Interventional Neuroradiology, First Affiliated Hospital of Zhengzhou University, Zhengzhou; ${ }^{4} \mathrm{Key}$ Laboratory of Psychiatric Disorders of Guangdong Province, Department of Neurobiology, School of Basic Medical Science, Southern Medical University, Guangzhou; and ${ }^{5}$ Department of Neurosurgery, Second Affiliated Hospital of Guangzhou Medical University, Guangzhou, China
\end{abstract}

OBJECTIVE Among clinical and morphological criteria, hemodynamics is the main predictor of aneurysm growth and rupture. This study aimed to identify which hemodynamic parameter in the parent artery could independently predict the rupture of anterior communicating artery $(\mathrm{ACOA})$ aneurysms by using multivariate logistic regression and two-piecewise linear regression models. An additional objective was to look for a more simplified and convenient alternative to the widely used computational fluid dynamics (CFD) techniques to detect wall shear stress (WSS) as a screening tool for predicting the risk of aneurysm rupture during the follow-up of patients who did not undergo embolization or surgery.

METHODS One hundred sixty-two patients harboring ACOA aneurysms (130 ruptured and 32 unruptured) confirmed by $3 \mathrm{D}$ digital subtraction angiography at three centers were selected for this study. Morphological and hemodynamic parameters were evaluated for significance with respect to aneurysm rupture. Local hemodynamic parameters were obtained by MR angiography and transcranial color-coded duplex sonography to calculate WSS magnitude. Multivariate logistic regression and a two-piecewise linear regression analysis were performed to identify which hemodynamic parameter independently characterizes the rupture status of $A C O A$ aneurysms.

RESULTS Univariate analysis showed that WSS $(p<0.001)$, circumferential wall tension $(p=0.005)$, age $(p<0.001)$, the angle between the $A_{1}$ and $A_{2}$ segments of the anterior cerebral artery $(p<0.001)$, size ratio $(p=0.023)$, aneurysm angle $(p<0.001)$, irregular shape $(p=0.005)$, and hypertension (grade II) $(p=0.006)$ were significant parameters. Multivariate analyses showed significant association between WSS in the parent artery and ACoA aneurysm rupture $(p=0.0001)$. WSS magnitude, evaluated by a two-piecewise linear regression model, was significantly correlated with the rupture of the ACoA aneurysm when the magnitude was higher than 12.3 dyne/cm² $(\mathrm{HR} 7.2,95 \% \mathrm{Cl} 1.5-33.6, p=$ 0.013).

CONCLUSIONS WSS in the parent artery may be one of the reliable hemodynamic parameters characterizing the rupture status of ACoA aneurysms when the WSS magnitude is higher than $12.3 \mathrm{dyne} / \mathrm{cm}^{2}$. Analysis showed that with each additional unit of WSS (even with a 1-unit increase of WSS), there was a 6.2-fold increase in the risk of rupture for ACoA aneurysms.

https://thejns.org/doi/abs/10.3171/2018.4.JNS173179

KEYWORDS anterior communicating artery aneurysm; risk of rupture; wall shear stress; hemodynamics; vascular disorders

ABBREVIATIONS ACA = anterior cerebral artery; $\mathrm{ACOA}=$ anterior communicating artery; $\mathrm{CFD}=$ computational fluid dynamics; $\mathrm{CWT}=$ circumferential wall tension; $\mathrm{DSA}=$ digital subtraction angiography; MRA = MR angiography; $\mathrm{SAH}=$ subarachnoid hemorrhage; TCCD = transcranial color-coded duplex; WSS = wall shear stress. SUBMITTED December 21, 2017. ACCEPTED April 26, 2018.

INCLUDE WHEN CITING Published online September 28, 2018; DOI: 10.3171/2018.4.JNS173179. 
$\mathrm{M}$ ORE often than not, it is difficult to predict intracranial aneurysm growth and rupture. ${ }^{35} \mathrm{Re}-$ cently, several studies of morphological and hemodynamic parameters have used numeral simulation of computational fluid dynamics (CFD) techniques and found that both high and low aneurysmal wall shear stress (WSS) have separately correlated with intracranial aneurysm growth and rupture. ${ }^{15,18,24,25}$ However, specific geometries with different aneurysm locations in each study may result in divergent and controversial findings..$^{22,35}$ The results of large-scale and multicenter clinical trials have been insufficient in clinical application. Additionally, few previous studies have evaluated WSS in the parent artery as being independently associated with the rupture of anterior communicating artery ( $\mathrm{ACoA})$ aneurysms using a more simplified and convenient alternative to the widely used CFD techniques. Although numeral simulation of CFD techniques has been widely used to detect WSS in aneurysms and to predict aneurysms' risk of rupture with encouraging results, it requires fluid dynamic technicians other than physicians for professional software analysis of CFD. It can be a complex process with a high cost in the majority of primary care facilities and community hospitals in China and many developing countries; thus, it is difficult to undertake in patients at each follow-up visit.

For the purpose of simple and accurate predictive assessment, 3D digital subtraction angiography (DSA), MR angiography (MRA), and transcranial color-coded duplex (TCCD) sonography techniques were used simultaneously to evaluate the characteristic morphology and identify the predictive factors of ACoA aneurysm rupture, using multivariate logistic regression and two-piecewise linear regression models; our intention was to look for a more simplified and convenient alternative to CFD techniques in the clinic to monitor WSS in the parent artery as a screening tool for predicting the risk of aneurysm rupture during the follow-up of patients who did not undergo embolization or surgery.

Significantly, this simple and accessible method is only appropriate for evaluating WSS in the parent artery, which is highly related to aneurysm rupture. However, it could gain popularity in many developing countries because of its relatively low use of resources.

\section{Methods \\ Patient Population}

Approval for this study was obtained from the local institutional review board of the participating centers. One hundred sixty-two patients harboring 130 ruptured and 32 unruptured ACoA aneurysms confirmed by 3D DSA were selected for this cross-sectional study at Southern Medical University Zhujiang Hospital, the Second Affiliated Hospital of Guangzhou Medical University, and the First Affiliated Hospital of Zhengzhou University, from September 2013 to July 2017. Cases involving multiple aneurysms were excluded from this study. Morphological and hemodynamic parameters were evaluated for significance with respect to aneurysm rupture.

The data were anonymous, and the requirement for informed consent was therefore waived.

\section{Evaluation of Morphological and Hemodynamic Characteristics}

Three-dimensional DSA was performed in all patients after admission. Morphological parameters were measured from 3D DSA images, including aneurysm parameters such as size, neck, shape, angle, aspect ratio, and size ratio. The cerebral vascular morphological structures, such as the dominant $A_{1}$ segment and the angle between the $A_{1}$ and $\mathrm{A}_{2}$ segments of the anterior cerebral artery (ACA), were also recorded. Hemodynamic parameters, including inner diameter at the end of diastolic blood pressure and mean blood flow velocity, were obtained using a 3.0T MRA scanner (Siemens) and TCCD sonography. Blood viscosity was measured in vitro at $37^{\circ} \mathrm{C}$ on the same day as the TCCD sonography examination with a cone/plate viscometer.

TCCD sonography was performed with a Diasonic VST Master Series. It contains a 2.0-MHz real-time imaging transducer and a $2.0-\mathrm{MHz}$ pulsed Doppler transducer. The maximum in situ Doppler energy output intensity was $89 \mathrm{~mW} / \mathrm{cm}^{2} \mathrm{I}_{\text {STPA.3 }}$ (spatial peak time average intensity). The ACA was imaged using a transtemporal window with the patient in the supine position. The mean blood flow velocity was measured for the bilateral distal end of the each $\mathrm{A}_{1}$ segment of the ACA. ${ }^{9}$ The ultrasound probe was placed as closely as possible to the distal end of each $\mathrm{A}_{1}$ segment, and the lateral and paramedian frontal bone windows were also used for accurate detection, according to the procedure described by Stolz et al. ${ }^{29}$ Each TCCD sonography examination was performed with the sample volume being placed within the color flow image of the examined artery.

MRA images were analyzed using a computer program (Flova; R'Tech) to evaluate the status of the flow around the ACA, including the ACoA wall, and the status of the aneurysm based on 3D-space bloodstream information. Additionally, the state of the bloodstream in the ACoA provided by cine phase-contrast MRI was also determined. ${ }^{20}$

In the case of aplasia of the $A_{1}$ segment, these measurements were applied only to the dominant $A_{1}$ segment. If the $A_{1}$ segments were symmetrical, the parameters were measured at the ipsilateral $A_{1}$ in aneurysm cases. The velocity of the ACA blood flow was examined by TCCD sonography 3 times. If the results were similar to those of MRA, the data were considered valid for inclusion; if the results differed significantly, the data were excluded from the study. Subsequently, the velocities of the ACoA blood flow were examined by TCCD sonography 3 times, and then the average was taken. For some patients, an accidental rise in WSS in the parent artery could presumably occur as part of normal daily blood pressure variance. If higher WSS is detected while the blood pressure rises, we suggest repeating the measurement after the participant has rested for at least 5-10 minutes.

Finally, WSS was calculated by Poiseuille's law according to the following formula: ${ }^{33} \mathrm{WSS}=4 \times \eta \times \mathrm{Vm} /$ inner diameter $\left(\right.$ dyne $\left./ \mathrm{cm}^{2}\right)$, where $\eta$ is blood viscosity and $\mathrm{Vm}$ is mean blood flow velocity.

Circumferential wall tension (CWT) was calculated by Laplace's law according to the following formula: ${ }^{10} \mathrm{CWT}$ $=$ mean blood pressure $\times($ inner diameter $/ 2)\left(\right.$ dyne $\left./ \mathrm{cm}^{2}\right)$. 
Note that WSS is defined as a flow-induced stress that can be described as the frictional force of viscous blood. ${ }^{16}$ CWT is a tensile stress divided by wall thickness, and it acts perpendicularly to the arterial wall and results from the extensional (dilating) effect of blood pressure on the vessel. ${ }^{1}$ The mean blood pressure was computed as diastolic blood pressure + one-third of the differential pressure.

\section{Statistical Analysis}

Continuous variables were expressed as mean \pm SD (normal distribution) or median (quartile) (skewed distribution). Categorical variables were expressed in frequency or as a percentage. The t-test (normal) or one-way ANOVA, Mann-Whitney U-test (skewed distribution), or KruskalWallis $\mathrm{H}$ and chi-square test (categorical variables) were used to determine any statistical differences between the means and proportions of the WSS groups. Multiple WSS models were used to evaluate the associations between exposure (WSS) and outcome (ACoA aneurysm rupture). Both nonadjusted and multivariate-adjusted models were used. A two-piecewise linear regression model was used to examine the threshold effect of the WSS on ACoA aneurysm rupture according to the smoothing plot. The threshold level of WSS at which the relationship between ACoA aneurysm rupture and WSS magnitude began to change and become notable was determined using a recurrence method. The inflection point was moved along a predefined interval until the inflection point that gave the maximum model likelihood was detected.

All of the analyses were performed with the statistical software packages R (http://www.R-project.org) and EmpowerStats (http://www.empowerstats.com). Statistical significance was set at $\mathrm{p}<0.05$ (two-sided).

\section{Results}

\section{Selection of Participants}

A total of 162 patients harboring $162 \mathrm{ACoA}$ aneurysms were selected, including 130 ruptured and 32 unruptured aneurysms. Detailed information is shown in Table 1.

\section{Baseline Characteristics of Participants}

The baseline characteristics of patients are also shown in Table 1. Continuous variables were examined by the Kruskal-Wallis rank-sum test. If the variable had a theoretical number less than 10 , the probability is calculated accurately using Fisher's exact test. In case of nonnormal distribution, variables were analyzed with the Wilcoxon rank-sum test. The statistical results showed that there was a statistically significant difference in age, sex, aspect ratio, CWT, and alcohol consumption among the categorical variable (quartile) groups of WSS.

\section{Univariate Analysis for Each Variable}

Univariate linear regression models were used to evaluate the associations between parameter variables and ACoA aneurysm rupture. The results of univariate analysis are shown in Table 2. Analysis demonstrated that WSS $(\mathrm{p}<0.001)$, CWT $(\mathrm{p}=0.005)$, age $(\mathrm{p}<0.001)$, angle be- tween the $\mathrm{A}_{1}$ and $\mathrm{A}_{2}$ segments of the ACA $(\mathrm{p}<0.001)$, size ratio $(p=0.023)$, aneurysm angle $(p<0.001)$, irregular shape $(p=0.005)$, and hypertension (grade II) $(p=0.006)$ were the risk factors associated with ACoA aneurysm rupture. In contrast, sex, aspect ratio, dominant $\mathrm{A}_{1}$ segment, aneurysm size, neck, presence of diabetes, atherosclerosis, coronary artery disease, smoking, and alcohol consumption were not associated with the event occurrence.

\section{Multivariate Analyses}

The WSS, which is the most significant hemodynamic parameter correlated with ACoA aneurysm rupture, was identified as a candidate variable for multivariate analyses.

Nonadjusted and adjusted models are illustrated in Table 3. In the crude model, WSS showed a correlation with ACoA aneurysm rupture (HR 1.4, 95\% CI 1.4-1.6, $\mathrm{p}<0.001$ ). In the minimally adjusted model (adjusted age, sex), the effect size also had no correlation (HR 1.4, 95\% CI $1.0-1.9, \mathrm{p}=0.055)$. After adjusting other covariates, we identified a correlation in the fully adjusted model (HR $1.3,95 \%$ CI $1.1-1.6, p=0.001$ ). For the purpose of sensitivity analysis, we also handled WSS as a categorical variable (quartile) and found that the same trend was observed as well $(\mathrm{p}=0.011)$.

\section{Results of the Two-Piecewise Linear Regression Model}

We used a two-piecewise linear regression model to examine the threshold effect of the WSS in the parent artery on ACoA aneurysm rupture, according to the smoothing plot. The threshold level of WSS at which the relationship between ACoA aneurysm rupture and WSS magnitude began to change and become notable was determined using a recurrence method. The inflection point was moved along a predefined interval until we detected the inflection point that gave the maximum model likelihood. The results of the two-piecewise linear regression model are shown in Table 4.

As evaluated by the two-piecewise linear regression model, the WSS magnitude is significantly correlated with rupture of ACoA aneurysms when it is higher than 12.3 dyne $/ \mathrm{cm}^{2}($ HR 7.2, 95\% CI 1.5-33.6, p = 0.013) (Table 4, Fig. 1). The model showed that with each additional unit of WSS (even with 1-unit increase of WSS), there was a 6.2fold increase in the risk of rupture for ACoA aneurysms.

\section{Discussion}

Our study showed that WSS, CWT, age, angle between the $A_{1}$ and $A_{2}$ segments of the ACA, size ratio, aneurysm angle, irregular shape, and hypertension (grade II) were significantly correlated with rupture status of ACoA aneurysms. WSS in the parent artery was identified as an independent risk factor for predicting the rupture status of ACoA aneurysms. Moreover, we found that WSS may be the most reliable hemodynamic parameter characterizing the rupture status of ACA aneurysms when it is higher than 12.3 dyne $/ \mathrm{cm}^{2}$ (HR 7.2, 95\% CI 1.5-33.6, $\mathrm{p}=0.013$ ). Using the two-piecewise linear regression model, we showed that with each additional unit of WSS (even with a 1-unit increase of WSS), there was a 6.2-fold increase in the risk of rupture of ACoA aneurysms. 
TABLE 1. Baseline characteristics of participants

\begin{tabular}{|c|c|c|c|c|c|c|}
\hline \multirow[b]{2}{*}{ Characteristic } & \multicolumn{4}{|c|}{ WSS Quartile } & \multirow{2}{*}{$\begin{array}{c}p \\
\text { Value }\end{array}$} & \multirow{2}{*}{$\begin{array}{c}p \\
\text { Value* }\end{array}$} \\
\hline & Q1 & Q2 & Q3 & Q4 & & \\
\hline No. of cases & 40 & 39 & 42 & 41 & & \\
\hline CWT (dyne/cm²) & $3.7 \pm 0.9$ & $3.9 \pm 1.0$ & $3.9 \pm 0.9$ & $3.2 \pm 1.1$ & 0.002 & $<0.001$ \\
\hline Age (yrs) & $61.3 \pm 10.4$ & $62.7 \pm 10.1$ & $61.6 \pm 8.1$ & $54.0 \pm 14.3$ & 0.002 & 0.007 \\
\hline Sex, no. & & & & & 0.033 & - \\
\hline Male & $25(62.5 \%)$ & $34(87.2 \%)$ & $34(81.0 \%)$ & $27(65.9 \%)$ & & \\
\hline Female & $15(37.5 \%)$ & $5(12.8 \%)$ & $8(19.0 \%)$ & $14(34.1 \%)$ & & \\
\hline Angle btwn $A_{1} \& A_{2}\left({ }^{\circ}\right) \dagger$ & $103.3 \pm 8.7$ & $105.8 \pm 6.7$ & $106.6 \pm 8.1$ & $107.9 \pm 9.4$ & 0.090 & 0.192 \\
\hline Aspect ratio† & $2.1 \pm 0.7$ & $2.5 \pm 0.8$ & $2.3 \pm 0.7$ & $2.6 \pm 0.8$ & 0.018 & 0.032 \\
\hline Size ratio† & $5.5 \pm 2.7$ & $5.0 \pm 2.3$ & $4.5 \pm 1.9$ & $4.7 \pm 2.0$ & 0.212 & 0.627 \\
\hline Aneurysm angle $\left(^{\circ}\right) \dagger$ & $133.4 \pm 26.1$ & $133.4 \pm 28.8$ & $133.6 \pm 27.6$ & $126.9 \pm 31.5$ & 0.654 & 0.822 \\
\hline Dominant $A_{1}$, no. & & & & & 0.064 & - \\
\hline No & $25(62.5 \%)$ & $32(82.1 \%)$ & $26(61.9 \%)$ & $33(80.5 \%)$ & & \\
\hline Yes & $15(37.5 \%)$ & $7(17.9 \%)$ & $16(38.1 \%)$ & $8(19.5 \%)$ & & \\
\hline Shape, no. & & & & & 0.081 & - \\
\hline Regular & $12(30.0 \%)$ & $20(51.3 \%)$ & $24(57.1 \%)$ & $18(43.9 \%)$ & & \\
\hline Irregular & $28(70.0 \%)$ & $19(48.7 \%)$ & $18(42.9 \%)$ & $23(56.1 \%)$ & & \\
\hline Size, no. & & & & & 0.990 & - \\
\hline$\leq 2 \mathrm{~mm}$ & $9(22.5 \%)$ & $9(23.1 \%)$ & $7(16.7 \%)$ & $7(17.1 \%)$ & & \\
\hline$>2, \leq 5 \mathrm{~mm}$ & $8(20.0 \%)$ & $8(20.5 \%)$ & $10(23.8 \%)$ & $8(19.5 \%)$ & & \\
\hline$>5, \leq 15 \mathrm{~mm}$ & $17(42.5 \%)$ & $17(43.6 \%)$ & $20(47.6 \%)$ & $22(53.7 \%)$ & & \\
\hline$>15 \mathrm{~mm}$ & $6(15.0 \%)$ & $5(12.8 \%)$ & $5(11.9 \%)$ & $4(9.8 \%)$ & & \\
\hline Neck & & & & & 0.142 & - \\
\hline Wide & $13(32.5 \%)$ & $5(12.8 \%)$ & $11(26.2 \%)$ & $7(17.1 \%)$ & & \\
\hline Narrow & $27(67.5 \%)$ & $34(87.2 \%)$ & $31(73.8 \%)$ & $34(82.9 \%)$ & & \\
\hline Hypertension, no. & & & & & 0.345 & - \\
\hline No & $24(60.0 \%)$ & $23(59.0 \%)$ & $26(61.9 \%)$ & $27(65.9 \%)$ & & \\
\hline Grade I & $4(10.0 \%)$ & $2(5.1 \%)$ & $6(14.3 \%)$ & $4(9.8 \%)$ & & \\
\hline Grade II & $12(30.0 \%)$ & $14(35.9 \%)$ & $10(23.8 \%)$ & $8(19.5 \%)$ & & \\
\hline Grade III & $0(0.0 \%)$ & $0(0.0 \%)$ & $0(0.0 \%)$ & $2(4.9 \%)$ & & \\
\hline Diabetes, no. & & & & & 0.122 & - \\
\hline No & $36(90.0 \%)$ & $35(89.7 \%)$ & $31(73.8 \%)$ & $32(78.0 \%)$ & & \\
\hline Yes & $4(10.0 \%)$ & $4(10.3 \%)$ & $11(26.2 \%)$ & $9(22.0 \%)$ & & \\
\hline Atherosclerosis, no. & & & & & 0.184 & - \\
\hline No & $36(90.0 \%)$ & $31(79.5 \%)$ & $31(73.8 \%)$ & $36(87.8 \%)$ & & \\
\hline Yes & $4(10.0 \%)$ & $8(20.5 \%)$ & $11(26.2 \%)$ & $5(12.2 \%)$ & & \\
\hline Alcohol consumer & & & & & 0.023 & - \\
\hline No & $38(95.0 \%)$ & $28(71.8 \%)$ & $30(71.4 \%)$ & $34(82.9 \%)$ & & \\
\hline Yes & $2(5.0 \%)$ & $11(28.2 \%)$ & $12(28.6 \%)$ & $7(17.1 \%)$ & & \\
\hline CAD, no. & & & & & 0.669 & - \\
\hline No & $38(95.0 \%)$ & $35(89.7 \%)$ & $40(95.2 \%)$ & $37(90.2 \%)$ & & \\
\hline Yes & $2(5.0 \%)$ & $4(10.3 \%)$ & $2(4.8 \%)$ & $4(9.8 \%)$ & & \\
\hline Smoking, no. & & & & & 0.118 & - \\
\hline No & $38(95.0 \%)$ & $33(84.6 \%)$ & $32(76.2 \%)$ & $33(80.5 \%)$ & & \\
\hline Yes & $2(5.0 \%)$ & $6(15.4 \%)$ & $10(23.8 \%)$ & $8(19.5 \%)$ & & \\
\hline
\end{tabular}

$\mathrm{CAD}=$ coronary artery disease.

* Continuous variables were obtained by the Kruskal-Wallis rank-sum test. If the count variable had a theoretical number $<10$, the probability was calculated accurately using Fisher's exact test.

$\dagger$ Values are presented as the mean \pm SD. 
TABLE 2. Results of univariate analysis

\begin{tabular}{|c|c|c|c|}
\hline \multirow[b]{2}{*}{ Variable } & \multirow[b]{2}{*}{ Value } & \multicolumn{2}{|c|}{ ACoA Aneurysm Rupture } \\
\hline & & OR $(95 \% \mathrm{Cl})$ & p Value \\
\hline WSS $^{*}$ & $9.0 \pm 2.9$ & $1.4(1.2-1.6)$ & $<0.001$ \\
\hline $\mathrm{CWT}^{*}$ & $3.7 \pm 1.0$ & $0.5(0.3-0.8)$ & 0.005 \\
\hline Age $(y r s)^{*}$ & $59.9 \pm 11.4$ & $0.9(0.9-1.0)$ & $<0.001$ \\
\hline \multicolumn{4}{|l|}{ Sex, no. } \\
\hline Male & $120(74.1 \%)$ & 1.0 & \\
\hline Female & $42(25.9 \%)$ & $2.0(0.9-4.6)$ & 0.099 \\
\hline Angle btwn $A_{1} \& A_{2}\left({ }^{\circ}\right)^{*}$ & $105.9 \pm 8.4$ & $1.1(1.1-1.2)$ & $<0.001$ \\
\hline Aspect ratio* & $2.4 \pm 0.8$ & $1.3(0.8-2.1)$ & 0.369 \\
\hline Size ratio* & $4.9 \pm 2.3$ & $0.8(0.6-1.0)$ & 0.023 \\
\hline Aneurysm angle $\left({ }^{\circ}\right)^{*}$ & $131.8 \pm 28.5$ & $1.0(1.0-1.0)$ & $<0.001$ \\
\hline \multicolumn{4}{|l|}{ Dominant $A_{1}$, no. } \\
\hline No & $116(71.6 \%)$ & 1.0 & \\
\hline Yes & $46(28.4 \%)$ & $1.2(0.5-2.8)$ & 0.690 \\
\hline \multicolumn{4}{|l|}{ Shape, no. } \\
\hline Regular & $74(45.7 \%)$ & 1.0 & \\
\hline Irregular & $88(54.3 \%)$ & $0.3(0.1-0.7)$ & 0.005 \\
\hline \multicolumn{4}{|l|}{ Size, no. } \\
\hline$\leq 2 \mathrm{~mm}$ & $32(19.8 \%)$ & 1.0 & \\
\hline$>2, \leq 5 \mathrm{~mm}$ & $34(21.0 \%)$ & $0.3(0.1-1.1)$ & 0.061 \\
\hline$>5, \leq 15 \mathrm{~mm}$ & $76(46.9 \%)$ & $0.6(0.2-1.5)$ & 0.260 \\
\hline$>15 \mathrm{~mm}$ & $20(12.3 \%)$ & $0.2(0.0-1.3)$ & 0.092 \\
\hline \multicolumn{4}{|l|}{ Neck } \\
\hline Wide & $36(22.2 \%)$ & 1.0 & \\
\hline Narrow & $126(77.8 \%)$ & $\operatorname{lnf}(0.0-\operatorname{lnf})$ & 0.987 \\
\hline \multicolumn{4}{|l|}{ Hypertension, no. } \\
\hline No & $100(61.7 \%)$ & 1.0 & \\
\hline Grade I & $16(9.9 \%)$ & $0.0(0.0-\operatorname{lnf})$ & 0.991 \\
\hline Grade II & $44(27.2 \%)$ & $0.1(0.0-0.5)$ & 0.006 \\
\hline Grade III & $2(1.2 \%)$ & $\operatorname{lnf}(0.0-\operatorname{lnf})$ & 0.997 \\
\hline \multicolumn{4}{|l|}{ Diabetes, no. } \\
\hline No & $134(82.7 \%)$ & 1.0 & \\
\hline Yes & $28(17.3 \%)$ & $0.9(0.3-2.5)$ & 0.782 \\
\hline \multicolumn{4}{|l|}{ Atherosclerosis, no. } \\
\hline No & $134(82.7 \%)$ & 1.0 & \\
\hline Yes & $28(17.3 \%)$ & $0.6(0.2-2.0)$ & 0.427 \\
\hline \multicolumn{4}{|l|}{ CAD, no. } \\
\hline No & $150(92.6 \%)$ & 1.0 & \\
\hline Yes & $12(7.4 \%)$ & $0.8(0.2-3.8)$ & 0.781 \\
\hline \multicolumn{4}{|l|}{ Smoking, no. } \\
\hline No & $136(84.0 \%)$ & 1.0 & \\
\hline Yes & $26(16.0 \%)$ & $1.6(0.6-4.3)$ & 0.320 \\
\hline \multicolumn{4}{|l|}{ Alcohol consumer, no. } \\
\hline No & $130(80.2 \%)$ & 1.0 & \\
\hline Yes & $32(19.8 \%)$ & $0.7(0.2-2.0)$ & 0.514 \\
\hline
\end{tabular}

Inf = infinity.

${ }^{*}$ Values are presented as the mean \pm SD.
Recently, image-based numeral simulation models of CFD have confirmed close correlations between hemodynamics and intracranial aneurysm growth and rupture, $7,14,15,18,19,22,24,25,27$ albeit with divergent and controversial findings regarding WSS. Meng et al. ${ }^{18}$ suggested that the high and low WSS threshold values vary separately in aneurysm development and remain unclear. The WSS is an extraordinarily complex mechanism composed of varied morphologies, flow dynamics, and genetics, which may also be affected by environmental contributing factors, whose interaction with hemodynamics has not been clearly elucidated. The morphological and hemodynamic parameters could modify the vascular response to both aneurysm growth and rupture.

More and more published CFD studies seem to suggest that high WSS results in intracranial aneurysm growth and low WSS in rupture. $7,12,14,15,18,19,22,24,25,27$ However, these results were inconsistent with those obtained by Liu et al. and Yu et al. 13,26,30,34 They found high WSS in the ruptured aneurysm group. Moreover, it is important to note that their average WSS in the aneurysm region $(1.64 \pm 1.16$ $\mathrm{N} / \mathrm{m}^{2}$ ) was significantly lower than that observed in the vessel region $(p<0.05)$, which highly accords with our findings. Other researchers addressed the relationship between WSS in the parent artery and aneurysm rupture.,23 Qiu et al. ${ }^{23}$ reported that ruptured aneurysms were more likely to have a higher aneurysm parent artery WSS ratio $(p=0.026)$ than unruptured aneurysms. They identified the highest aneurysm parent artery WSS ratios $(p=0.023)$ as the hemodynamic factors predictive of intracranial aneurysm rupture.

It is widely accepted that arteries adapt to blood flow changes through vascular remodeling of themselves. WSS arising from friction of blood flow against the vessel wall is an essential determinant of vascular remodeling. These changes in WSS have been investigated in several studies, in both humans and animals. ${ }^{8,17}$ Physiologically, the endothelium actively assists in the maintenance of normal levels of WSS by using various compensatory mechanisms. In pathological conditions, vasospasm occurs rapidly following flow alteration, when the aneurysm ruptures. WSS acts on the endothelium and is the mechanical force responsible for the acute change in luminal diameter. ${ }^{8,32}$ This biological response is achieved primarily by the vasodilators released from smooth muscle cells, ultimately causing the vessel changes. ${ }^{32}$ Hoi et al. ${ }^{8}$ demonstrated that the baseline WSS level is $10-15 \mathrm{dyne} / \mathrm{cm}^{2}$ in all arteries. There exists a flow-increase threshold (approximate 25\%-140\% increase in baseline WSS). When it is surpassed, high WSS may result in significant vascular tortuosity. Accordantly, what our study showed supports this view. We discovered that high WSS in the parent artery was significantly correlated with ACoA aneurysm rupture when it was higher than 12.3 dyne $/ \mathrm{cm}^{2}$, which is partially inconsistent with the high- or low-WSS theory. $7,14,15,18,19$, 22,24,25,27 WSS in the parent artery may be different from that in an ACoA aneurysm itself. Vasospasm caused by subarachnoid hemorrhage (SAH) when aneurysms easily rupture results in a smaller inner diameter and increased blood flow velocity. ${ }^{4,11,28}$ As a result, the WSS, calculated by formulas according to Poiseuille's law, would also in- 
TABLE 3. Results of multivariate analysis

\begin{tabular}{cccc}
\hline & \multicolumn{3}{c}{ Hazard Ratio $(95 \% \mathrm{Cl}), \mathrm{p}$ Value } \\
\cline { 2 - 4 } Exposure & Crude Model $^{*}$ & Minimally Adjusted Model† & Fully Adjusted Modelf \\
\hline WSS & $1.4(1.2-1.6),<0.001$ & $1.4(1.0-1.9), 0.055$ & $1.3(1.1-1.6), 0.001$ \\
\hline WSS quartile & & 1.0 & 1.0 \\
\hline Q1 & 1.0 & $0.1(0.0-1.8), 0.131$ & $0.6(0.1-2.7), 0.541$ \\
\hline Q2 & $0.8(0.2-3.2), 0.754$ & $0.1(0.0-2.4), 0.162$ & $0.7(0.2-2.8), 0.588$ \\
\hline Q3 & $0.9(0.3-3.6), 0.934$ & $2.8(0.2-31.3), 0.407$ & $0.2(1.3-14.0), 0.020$ \\
\hline Q4 & $5.5(1.8-16.8), 0.003$ & 0.334 & 0.011 \\
\hline p value for trend & 0.001 & & \\
\hline
\end{tabular}

crease. Thus, it is reasonable that high WSS in the parent artery is obtained when an aneurysm ruptures.

It has been reported that some structural changes after the aneurysm ruptures may affect the hemodynamic parameters. Thus, the hemodynamic features in a ruptured aneurysm may differ from those seen before the rupture. ${ }^{3,19}$ Focally identified hemosiderin staining around supposedly unruptured aneurysms has been observed during intracranial aneurysm clipping, suggesting instability. ${ }^{21}$ Furthermore, and more remarkably, this may indicate that there is a subgroup of individuals at increased risk of future aneurysmal SAH. ${ }^{5,31}$ During this unstable period, the WSS magnitude may exhibit subtle changes before aneurysm rupture. Since the WSS in ACAs may be greatly related to the rupture of ACoA aneurysms, it is very important to monitor the changes in WSS during follow-up examination of patients who did not undergo embolization or surgery for various reasons. If during such follow-up visits the WSS exhibits the trend of increasing, and even rising up to the threshold level we identified in the present study, early intervention should be strongly recommended to prevent any adverse events.

Studies with accurate WSS findings have not yet been reported for the use of parameters other than CFD techniques, such as clinical tests and conventional methods, in low-resource hospitals. Our simple and accessible method can be used to detect the WSS in modest primary care facilities and community hospitals in China and many developing countries. Although unable to detect direct WSS in aneurysms, it can be used to evaluate the WSS of the parent artery, which is highly related to aneurysm rupture.

Additionally, it is important to note that in the contro-

TABLE 4. Results of the two-piecewise linear regression model

\begin{tabular}{cccc}
\hline Inflection Point of WSS & Effect Size $(\mathrm{HR})$ & $95 \% \mathrm{Cl}$ & $\mathrm{p}$ Value \\
\hline$\leq 12.3$ & 0.8 & $0.6-1.2$ & 0.353 \\
\hline$>12.3$ & 7.2 & $1.5-33.6$ & 0.013 \\
\hline
\end{tabular}

Fully adjusted model adjusted for CWT, age, sex, angle between $A_{1}$ and $A_{2}$ segments, aspect ratio, size ratio, aneurysm angle, dominant $A_{1}$ segment, shape, size, neck, hypertension, diabetes, atherosclerosis, coronary artery disease, smoking, and alcohol consumption. versial parameters of high versus low WSS, multivariate analysis was usually performed..$^{6,14,19,36}$ However, few researchers have further studied the threshold effect of WSS on ACoA aneurysm rupture using the two-piecewise linear regression model. In our cross-sectional study, univariate linear regression models and multivariate analyses were used to predict the risk of ACoA aneurysm rupture based on WSS in the parent artery. Additionally, the twopiecewise linear regression model revealed a more explicit meaning of WSS in a certain range.

Our study has some limitations. First, as this is a crosssectional study of correlation between WSS in the parent artery and ACoA aneurysm rupture, there is uncertainty in the degree to which exposure preceded the outcomes observed. Second, factors related to procedural detection may affect the accuracy of the results. Third, we measured only time-averaged WSS in the parent artery rather than that in the ACoA aneurysm itself. The direct WSS in ACoA aneurysms was not detectable using our clinical method. Though the result was different from that obtained when WSS was measured with the CFD technique, it is still an objective indicator of WSS in the parent artery. Fourth, intracranial aneurysms in different locations with varied morphologies and flow dynamics may have differing WSS values. This was also associated with complex genetic and environmental contributing factors. Thus, our findings may not characterize all kinds of intracranial aneurysms in different locations. Finally, some structural changes after the aneurysm rupture may affect the hemodynamic parameters. Thus, the hemodynamic features of ruptured aneurysms may differ from those recorded before the rupture. Unruptured aneurysms undergo microhemorrhages that may indicate instability, suggesting increased risk for aneurysmal SAH in the future. During this unstable period, the WSS magnitude may exhibit subtle changes before aneurysm rupture. Thus, we may be able to predict the risk of aneurysm rupture by monitoring the changes in WSS in the parent artery during follow-up for patients who did not undergo embolization or surgery. Our findings are just the tip of the iceberg. We hope that this paper provides valuable information and thus inspires valuable ideas for other researchers who are interested in this field. 


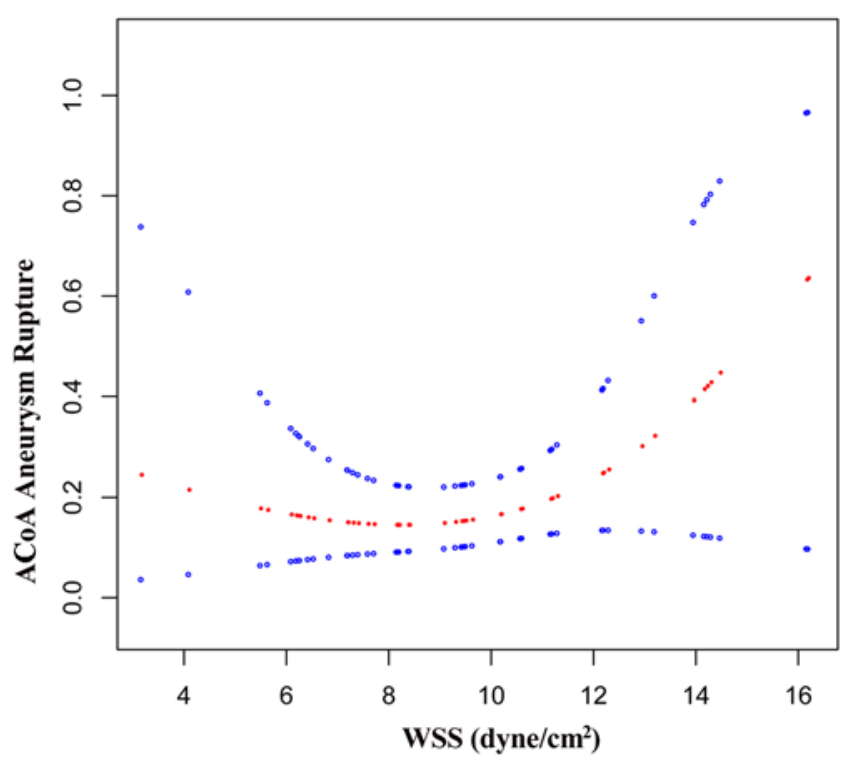

FIG. 1. Relation between WSS in the parent artery and ACoA aneurysm rupture. The area between two blue dotted lines represents the $95 \% \mathrm{Cl}$, and each red point shows the WSS magnitude in the parent artery, and the dots are connected to form a dotted line. The WSS magnitude is not correlated with ACoA aneurysm rupture when it is $\leq 12.3 \mathrm{dyne} / \mathrm{cm}^{2}$. Conversely, the WSS magnitude $>12.3$ dyne $/ \mathrm{cm}^{2}$ showed a significant correlation with $\mathrm{ACOA}$ aneurysm rupture. The risk of $\mathrm{ACOA}$ aneurysm rupture increases as the WSS increases. Figure is available in color online only.

\section{Conclusions}

WSS in the parent artery may be a reliable hemodynamic parameter to characterize the rupture status of ACoA aneurysms when it is higher than 12.3 dyne $/ \mathrm{cm}^{2}$. We found that with each additional unit of WSS (even with a 1-unit increase of WSS), there was a 6.2-fold increase in the risk of rupture for ACoA aneurysms.

Our simple and accessible method can provide objective evidence for predicting the risk of aneurysm rupture and can serve as a screening tool at follow-up for patients who did not receive embolization or surgery.

\section{Acknowledgments}

We would like to thank professor Chi Chen for his great contribution to the professional statistical analysis of the study data.

This work was supported by the National Key Research Development Program (grants 2016YFC1300804 and 2016YFC1300800), Science and Technology Project Foundation of Guangdong Province (grant 2016A020215098), and Key Project of Clinical Research of Southern Medical University (grant LC2016ZD024).

\section{References}

1. Carallo C, Irace C, Pujia A, De Franceschi MS, Crescenzo A, Motti C, et al: Evaluation of common carotid hemodynamic forces. Relations with wall thickening. Hypertension 34:217-221, 1999

2. Chien A, Tateshima S, Sayre J, Castro M, Cebral J, Viñuela F: Patient-specific hemodynamic analysis of small internal carotid artery-ophthalmic artery aneurysms. Surg Neurol 72:444-450, 2009

3. Doddasomayajula R, Chung BJ, Mut F, Jimenez CM,
Hamzei-Sichani F, Putman CM, et al: Hemodynamic characteristics of ruptured and unruptured multiple aneurysms at mirror and ipsilateral locations. AJNR Am J Neuroradiol 38:2301-2307, 2017

4. Duan Y, Wright J, Wright C, Shammassian B, Tatsuoka C, Bambakidis N: Reliable identification of benign clinical course in aneurysmal subarachnoid hemorrhage: a simple and qualitative algorithm. Neurosurgery [epub ahead of print], 2017

5. Falter B, Wiesmann M, Freiherr J, Nikoubashman O, Mull M: Frequency and appearance of hemosiderin depositions after aneurysmal subarachnoid hemorrhage treated by endovascular therapy. Neuroradiology 57:999-1006, 2015

6. Frösen J: Flow dynamics of aneurysm growth and rupture: challenges for the development of computational flow dynamics as a diagnostic tool to detect rupture-prone aneurysms. Acta Neurochir Suppl 123:89-95, 2016

7. Fukazawa K, Ishida F, Umeda Y, Miura Y, Shimosaka S, Matsushima S, et al: Using computational fluid dynamics analysis to characterize local hemodynamic features of middle cerebral artery aneurysm rupture points. World Neurosurg 83:80-86, 2015

8. Hoi Y, Gao L, Tremmel M, Paluch RA, Siddiqui AH, Meng $\mathrm{H}$, et al: In vivo assessment of rapid cerebrovascular morphological adaptation following acute blood flow increase. J Neurosurg 109:1141-1147, 2008

9. Irace C, Carallo C, De Franceschi MS, Scicchitano F, Milano M, Tripolino C, et al: Human common carotid wall shear stress as a function of age and gender: a 12-year follow-up study. Age (Dordr) 34:1553-1562, 2012

10. Jiang Y, Kohara K, Hiwada K: Association between risk factors for atherosclerosis and mechanical forces in carotid artery. Stroke 31:2319-2324, 2000

11. Ju C, Wright CH, Wright J, Duan Y, Bambakidis NC: Subarachnoid hemorrhage associated with intratumoral aneurysm rupture within a posterior fossa hemangioblastoma: the importance of continued surveillance for cerebral vasospasm. Cureus 9:e1606, 2017

12. Kawaguchi T, Nishimura S, Kanamori M, Takazawa H, Omodaka S, Sato K, et al: Distinctive flow pattern of wall shear stress and oscillatory shear index: similarity and dissimilarity in ruptured and unruptured cerebral aneurysm blebs. J Neurosurg 117:774-780, 2012

13. Liu J, Xiang J, Zhang Y, Wang Y, Li H, Meng H, et al: Morphologic and hemodynamic analysis of paraclinoid aneurysms: ruptured versus unruptured. J Neurointerv Surg 6:658-663, 2014

14. Longo M, Granata F, Racchiusa S, Mormina E, Grasso G, Longo GM, et al: Role of hemodynamic forces in unruptured intracranial aneurysms: an overview of a complex scenario. World Neurosurg 105:632-642, 2017

15. Lv N, Wang C, Karmonik C, Fang Y, Xu J, Yu Y, et al: Morphological and hemodynamic discriminators for rupture status in posterior communicating artery aneurysms. PLoS One 11:e0149906, 2016

16. Malek AM, Alper SL, Izumo S: Hemodynamic shear stress and its role in atherosclerosis. JAMA 282:2035-2042, 1999

17. Meng H, Metaxa E, Gao L, Liaw N, Natarajan SK, Swartz DD, et al: Progressive aneurysm development following hemodynamic insult. J Neurosurg 114:1095-1103, 2011

18. Meng H, Tutino VM, Xiang J, Siddiqui A: High WSS or low WSS? Complex interactions of hemodynamics with intracranial aneurysm initiation, growth, and rupture: toward a unifying hypothesis. AJNR Am J Neuroradiol 35:1254-1262, 2014

19. Miura Y, Ishida F, Umeda Y, Tanemura H, Suzuki H, Matsushima S, et al: Low wall shear stress is independently associated with the rupture status of middle cerebral artery aneurysms. Stroke 44:519-521, 2013 
20. Naito T, Miyachi S, Matsubara N, Isoda H, Izumi T, Haraguchi K, et al: Magnetic resonance fluid dynamics for intracranial aneurysms - comparison with computed fluid dynamics. Acta Neurochir (Wien) 154:993-1001, 2012

21. Nussbaum ES, Defillo A, Zelensky A, Pulivarthi S, Nussbaum L: "Microbleeding" from intracranial aneurysms: local hemosiderin deposition identified during microsurgical treatment of unruptured intracranial aneurysms. Surg Neurol Int 5:28, 2014

22. Qin H, Yang Q, Zhuang Q, Long J, Yang F, Zhang H: Morphological and hemodynamic parameters for middle cerebral artery bifurcation aneurysm rupture risk assessment. J Korean Neurosurg Soc 60:504-510, 2017

23. Qiu T, Jin G, Xing H, Lu H: Association between hemodynamics, morphology, and rupture risk of intracranial aneurysms: a computational fluid modeling study. Neurol Sci 38:1009-1018, 2017

24. Russell JH, Kelson N, Barry M, Pearcy M, Fletcher DF, Winter CD: Computational fluid dynamic analysis of intracranial aneurysmal bleb formation. Neurosurgery 73:1061-1069, 2013

25. Sejkorová A, Dennis KD, Švihlová H, Petr O, Lanzino G, Hejčl A, et al: Hemodynamic changes in a middle cerebral artery aneurysm at follow-up times before and after its rupture: a case report and a review of the literature. Neurosurg Rev 40:329-338, 2017

26. Shojima M, Oshima M, Takagi K, Torii R, Hayakawa M, Katada K, et al: Magnitude and role of wall shear stress on cerebral aneurysm: computational fluid dynamic study of 20 middle cerebral artery aneurysms. Stroke 35:2500-2505, 2004

27. Skodvin TØ, Evju Ø, Helland CA, Isaksen JG: Rupture prediction of intracranial aneurysms: a nationwide matched case-control study of hemodynamics at the time of diagnosis. J Neurosurg [epub ahead of print November 3, 2017; DOI: 10.3171/2017.5.JNS17195]

28. Sokolowski JD, Chen CJ, Ding D, Buell TJ, Raper DM, Ironside N, et al: Endovascular treatment for cerebral vasospasm following aneurysmal subarachnoid hemorrhage: predictors of outcome and retreatment. J Neurointerv Surg 10:367374, 2018

29. Stolz E, Kaps M, Kern A, Dorndorf W: Frontal bone windows for transcranial color-coded duplex sonography. Stroke 30:814-820, 1999

30. Sugiyama S, Meng H, Funamoto K, Inoue T, Fujimura M, Nakayama T, et al: Hemodynamic analysis of growing intracranial aneurysms arising from a posterior inferior cerebellar artery. World Neurosurg 78:462-468, 2012
31. Takada S, Inoue T, Niizuma K, Shimizu H, Tominaga T: Hemosiderin detected by $\mathrm{T} 2 *$-weighted magnetic resonance imaging in patients with unruptured cerebral aneurysms: indication of previous bleeding? Neurol Med Chir (Tokyo) 51:275-281, 2011

32. Tronc F, Mallat Z, Lehoux S, Wassef M, Esposito B, Tedgui A: Role of matrix metalloproteinases in blood flow-induced arterial enlargement: interaction with NO. Arterioscler Thromb Vasc Biol 20:E120-E126, 2000

33. Wang C, Chen M, Liu SL, Liu Y, Jin JM, Zhang YH: Spatial distribution of wall shear stress in common carotid artery by color Doppler flow imaging. J Digit Imaging 26:466-471, 2013

34. Yu Y, Xu J, Fang Y, Wu X, Yang P, Jiang C, et al: Analysis of morphologic and hemodynamic parameters for unruptured posterior communicating artery aneurysms with oculomotor nerve palsy. AJNR Am J Neuroradiol 34:2187-2191, 2013

35. Zhang X, Long XA, Luo B, Karuna T, Duan CZ: Factors responsible for poor outcome after intraprocedural rerupture of ruptured intracranial aneurysms: identification of risk factors, prevention and management on 18 cases. Eur J Radiol 81:e77-e85, 2012

36. Zhang Y, Tian Z, Jing L, Zhang Y, Liu J, Yang X: Bifurcation type and larger low shear area are associated with rupture status of very small intracranial aneurysms. Front Neurol 7:169, 2016

\section{Disclosures}

The authors report no conflict of interest concerning the materials or methods used in this study or the findings specified in this paper.

\section{Author Contributions}

Conception and design: Duan, Zhang. Acquisition of data: Duan, Zhang, Karuna, Yao, Jiang, X Li, Yin, He, Guo, Chen, Liu, R Li, Fan. Drafting the article: Zhang. Reviewed submitted version of manuscript: Zhang, Karuna. Approved the final version of the manuscript on behalf of all authors: Duan. Study supervision: Duan, Wang.

\section{Correspondence}

Chuan-Zhi Duan: Southern Medical University, Zhujiang Hospital, Guangdong, China.doctor_duanzj@163.com. 